\title{
OSAS In Young and Older Children: A Brief Review on the Role of Laryngomalacia
}

\author{
Gallucci Marcella, Masetti Riccardo, Cipriani Francesca, Giannetti Arianna, Bertelli Luca, \\ Pession Andrea and Ricci Giampaolo* \\ Pediatric Unit, Department of Medical and Surgical Sciences, S Orsola Malpighi University Hospital, Bologna, Italy \\ *Corresponding author: Ricci Giampaolo, Department of Pediatrics, S Orsola-Malpighi Hospital, University of Bologna, Bologna, \\ Italy
}

\section{ARTICLE INFO}

Received: 慧 February 23, 2020

Published: 椚 March 05, 2020

Citation: Gallucci Marcella, Masetti Riccardo, Cipriani Francesca, Giannetti Arianna, Bertelli Luca, Pession Andrea and Ricci Giampaolo. OSAS In Young and Older Children: A Brief Review on the Role of Laryngomalacia. Biomed J Sci \& Tech Res 26(2)-2020. BJSTR. MS.ID.004331.

\begin{abstract}
Obstructive Sleep Apnea Syndrome [OSAS] represent an emerging disease with a prevalence that range from $1 \%$ to $4 \%$. Common risk factor in children are adeno-tonsillar hypertrophy followed by other conditions as neuromuscular disorders, craniofacial anomalies and obesity. Among young children the presence of laryngomalacia can worsen respiratory symptoms especially in the first year of life. Congenital laryngomalacia is a common cause of stridor in infants and sometimes can results in failure to thrive, feeding difficult, aspiration and obstructive disease during sleep. It can be detected trough flexible fiberoptic laryngoscopy performed both in awake child and under sedation. Laryngomalacia that appears only during sleep is defined "state dependent" laryngomalacia. In this brief review we discuss the association of LM with OSAS among infants and older children, with particular attention to available diagnostic tools and the current therapeutic options comprising both medical and surgical approaches.
\end{abstract}

Keywords: Laryngomalacia; OSAS; Flexible Laryngoscopy; Drug Induced Sleep Endoscopy; CPAP; Supraglottoplasty

Abbreviations: LM: laryngomalacia; FFL: Flexible Fiberoptic laryngoscopy; OSAS: Obstructive Sleep Apnea Syndrome; GER: Gastroesophageal Reflux; DISE: Drug Induced Sleep Endoscopy; OAHI: Obstructive Apnea-Hypopnea Index; CPAP: Continuous Positive Airway Pressure

\section{Introduction}

Obstructive Sleep Apnea Syndrome [OSAS] is a common disease that reaches prevalence of $4 \%$ during childhood. Most common risk factors in children are adeno-tonsillar hypertrophy, neuromuscular disorders, craniofacial abnormalities and obesity. Nevertheless, among young children and infants' other conditions should be taken into account, including laryngomalacia, although few studies have investigated the larynx as the origin site of sleep apnea. The coexistence of laryngomalacia and OSAS is frequently overlooked. Laryngomalacia resolves spontaneously approximately in up to $80 \%$ of infants, but in the remaining cases some therapeutic approach is required [1]. Congenital laryngomalacia is a common cause of stridor in infants. Stridor is caused by a collapse of supraglottic structures during inspiration and in more serious cases this condition can cause failure to thrive, feeding difficult, aspiration and obstructive disordered during sleep. Stridor usually appears after first weeks of life and often increase during feeding, in supine position or in restlessness. The pathogenesis of laryngomalacia seems to be related to immaturity of laryngeal cartilages or abnormal sensorimotor integration that induce an inspiratory collapse of supraglottic structures [1,2]. Gastroesophageal reflux [GER] is frequently detected in association with LM. Several evidences suggested that the mucosal inflammation GER related worsens LM symptoms' while the negative intrathoracic pressure generated to overcome airway obstruction may predispose to GER in a vicious circle [2-4]. A study of Hadfield et coll. demonstrated that the surgical correction of laryngomalacia also improved GER as assessed by 24 -h probe monitoring [5]. LM frequently is associated 
with congenital or acquired neuromuscular disorders with a prevalence that widely range from 8 and 50\% [6]. Children with Down Syndrome have a high prevalence of airway obstruction at laryngeal and tracheal levels, particularly trachea-bronchomalacia. Laryngomalacia is often described as being common in children with Down's syndrome, where is generally correlated to the hypotonia and gastro-esophageal reflux disease $[7,8]$.

The incidence of OSAS in infants with laryngomalacia [LM] is unknown, although obstructive apnea can be a sign of severity of laryngomalacia [1,9]. A report conducted by Zalzal, et al. found that $80 \%$ of patients showed apneic events prior to treatment of supraglottoplasty. Other literature data on severe laryngomalacia described a similar picture, with an incidence of apnea ranging from 62 to $82 \%$ [10-12].The main diagnostic tool for the assessment of LM is the flexible fiberoptic laryngoscopy [FFL] that in small children is generally performed under general anesthesia. To ensure the maintenance of a spontaneous breathing, inhalation anesthetics should be used $[13,14]$. The gold standard to diagnose OSAS for all ages is the standard polysomnography [PSG]. Since physiologic polysomnographic parameters widely vary with age, age-appropriate norms should be used according to recent AASM guidelines $[15,16]$. Sometimes laryngomalacia may contribute to OSAS also in older children [17]. Isaacson et al. in 1997 defined "state-dependent laryngomalacia [SDL]" as a condition that appear during sleep with stridor and obstructive respiratory disorders in a child who present normal breathing while awake [18].

It is unclear whether this type of laryngomalacia is a continuum of a congenital disease or rather a condition that independently develops due to both redundant mucosae prolapsing over the arytenoids upon inspiration and neuromuscular abnormalities [19]. Typically, children present with sleep-induced stridor, upper airway obstruction and apnea. Sleep-state dependent laryngomalacia is estimated to cause upper airway obstruction in $3.9 \%$ of children with OSA assessed prior to adenotonsillectomy [20]. A study of Goldberg et al. described 17 subjects with sleeponset LM; among these children 7 had underlying hypotonia and 10 showed LM associated with OSA. Why these patients have a normalappearing laryngeal examination when awake but LM when asleep is unclear [21].

\section{Classification}

Several classifications of congenital laryngomalacia have been proposed, often based on anatomic abnormalities or dynamic changes observed during flexible laryngoscopy, although to date no codified classification system is accepted. Some static findings include omega-shaped epiglottis, acute angle of epiglottis (Holinger, et al.) and short aryepiglottic folds even though these anomalies do not consider the dynamic changes during spontaneous breathing [22]. The simplest used categorization, proposed by Olney and coll. includes the three following groups: type 1- anterior prolapse of the mucosa overlying the arytenoid cartilages; type 2- medial collapse of short aryepiglottic folds; type 3- posterior displacement of the epiglottis. Most subjects present with more than one type of obstruction [23]. Moreover, different combinations of the various types can be observed and often LM is associated with other airways lesions such as tracheomalacia, bronchomalacia, subglottic stenosis, and vocal cord paralysis [24].More recently Groningen et al. proposed a new classification comprising three types of laryngomalacia, all based on dynamic laryngeal findings observed during fiberoptic laryngoscopy:

a) Type 1: inward, antero-caudal collapse of arytenoids cartilages.

b) Type 2: medial displacement of aryepiglottic fold during inspiration.

c) Type 3: postero-caudal displacement of epiglottis against the posterior pharyngeal wall.

This classification provides a more didactic categorization of laryngomalacia and may be useful to guide the surgical approach [25].

\section{Diagnostic Tools}

The flexible fiberoptic laryngoscopy [FFL] is considered the gold standard for the detection of LM. It can be performed on the awake infant in upright position and often it allows excellent visualization of the supraglottic airway, allowing to visualize the location and degree of supraglottic obstruction. The inability to view the vocal folds on FFL may serve as an indication of severe disease [10]. When performed under sedation FFL should ensure the maintenance of spontaneous breathing so that the endoscopic findings were not distorted. Nevertheless, FFL in conscious infants may miss cases of sleep-induced laryngomalacia and sometimes its execution can be very difficult due to agitation's child. A study of Sivan et coll. showed a risk of false-negative rate of about $8 \%$ when laryngoscopy was performed in wake vs general anesthesia [26]. Among children $<2$ years with OSAS, the pattern of upper airway obstruction is poorly known due to lacking of data often derived by retrospective studies. When a "state dependent" LM is suspected, a useful diagnostic tool may be the drug induced sleep endoscopy [DISE] that consist of the upper airway inspection during induced sleep through light general anesthesia.

DISE provides a global evaluation of sites of obstruction during sedation and therefore can identify the level and the severity of collapse $[27,28]$. This technique is frequently used to assess children with complex upper airway disorders. Among children with OSAS, the execution of sleep endoscopy may help to plane the best surgical approach, even though a standardized method of interpretation that ensure an acceptable agreement among operators is lacking. DISE can be also indicated for children 
who have persistent OSA after adenotonsillectomy or for older children with OSA but without tonsillar hypertrophy when a state dependent laryngomalacia is suspected [29]. A retrospective study of Boudewyns and coll. involving 28 two-year-old children, showed a finding of collapse of epiglottis in 6 patients and laryngomalacia in others 4 subjects. All the children had severe OSAS. Among these children, DISE was useful in surgical decision making in order to achieve a targeted treatment based on the site of obstruction [30].

\section{Therapeutic Approach}

Treatment of OSAS associated with laryngomalacia may include conservative management [such as wait and see approach, antireflux treatment and CPAP ventilation] or surgery [summarized under the term of supraglottoplasty] that can be made urgently or in a second time. Supraglottoplasty is the current surgery of choice for severe LM. It includes the anatomically subdivided procedures of epiglottoplasty, aryepiglottoplasty, and arytenoidoplasty [31]. Summarily, it consists in the surgical removal of the redundant supraglottic mucosa guided by the findings during sleep endoscopy. Usually this procedure is performed under general anesthesia, with the patientspontaneouslybreathing, although sometimes intubation may be necessary $[32,33]$. When indicated, supraglottoplasty can be an effective procedure and may significantly improve symptoms of OSAS. A more effective treatment requires an accurate diagnosis that can be achieved during flexible laryngo-bronchoscopy with the child breathing spontaneously. Digoy and coll. reported a large series of children who were diagnosed with SDL and sleep apnea and who underwent a laser supraglottoplasty.33/36 patients experienced a statistically significant improvement in AHI/OAI after supraglottoplasty [19]. For less severe cases of LM, nonsurgical therapeutic strategies can be used, including non-invasive ventilation. Nasal CPAP may be a useful approach in children with OSAS and LM when upper airway obstruction is not resolved by adenotonsillectomy [34].The CPAP mechanism of action is due to a pneumatic thrust resulting in increase of the upper airways pressure that overcome the critical transmural pressure of the pharynx and the hypopharynx and prevent the airway collapse and obstructive events. The nasal mask should adhere to the child's face to avoid air leaks.

Some evidences showed that also among cases of severe laryngomalacia and vocal cord paralysis with OSA, non-invasive ventilation through nasal CPAP can be an effective and safe alternative to surgery [35].The CPAP titration usually starts with low pressure [4 $\mathrm{cm} \mathrm{H2O}$ ] gradually increased until obstructive sleep events and oxyhemoglobin desaturations are overcome. Monitoring of CPAP assessment is recommended every 6-12 months, since mask size and CPAP pressure should be modified according to child growth $[36,37]$.However, this treatment is not always accessible, since a specialized medical/nursing staff and an adequate training program for parents are both essential for the CPAP success. To date, little evidences are available regarding the indications for polysomnography and others sleep studies in children with laryngomalacia. Recent guidelines suggest that polysomnography should guide therapeutic approach only in infants with laryngomalacia associated with cardiac/neurological diseases or complex malformation syndrome [1].

Nevertheless, we believe that in all cases of assessed laryngomalacia it is prudent searching for obstructive sleep disorders since the prevalence of SDB among these children could be more frequent than expected. Frequently LM is associated with some endoscopic findings suggestive of reflux arytenoid and posterior glottic edema, vocal fold edema and lingual tonsil hypertrophy [38]. A systematic review regarding the association between laryngomalacia and reflux and comprising a meta-analysis on 6 studies, showed a prevalence of $59 \%$ based on dual probe $\mathrm{pH}$ monitoring although this was not statistically significantly greater in laryngomalacia than in other respiratory diagnoses. Response to anti-reflux therapy showed weak evidence, and benefit was generally apparent only after several months of therapy. Moreover, the histological findings showed only mild laryngeal inflammation. The authors concluded that any causal association between LM and GER lacks an evidence base therefore randomized controlled trial of anti-reflux therapy against placebo would be needed [39]. The International Pediatric Otorhinolaryngology Group consensus suggest an empirical approach recommending use of anti-reflux therapy if the child has feeding and/or respiratory problems, and 'only observation' if respiratory symptoms are mild and the growth is satisfactory [40].

\section{Discussion}

OSAS is an emerging disease that can cause significant morbidity at any age. Numerous studies suggest that SDB has a deleterious effect on infant's growth and intellectual development [9]. Infants with prolonged apnea often show lethargy, feeding difficulty, grown and developmental delay [41]. OSAS risk factors in infants differ from those of older children. Nevertheless, in early life as in older children, the presence of laryngomalacia can cause respiratory disorders requiring in the most severe cases some treatment. The real incidence of concurrent OSA and laryngomalacia is probably underestimated. Among older children with Obstructive Sleep Apnea Syndrome and daytime behavioral disturbances, laryngomalacia incidence has been reported to be 3.9\% [20]. Several studies have shown that from $20-75 \%$ of children may have some degree of persistent sleep disordered breathing after adenotonsillectomy. A picture of occult laryngomalacia has been identified as a reason for poor post-operative PSG improvement in patients with OSA that underwent adenotonsillectomy [42]. Described variants from the typical stridor that develops in infants include episodic, exercise induced, and late-onset laryngomalacia and tracheomalacia. The concomitant presence of neurological 
disorders and other co-morbidities could worsen symptoms. A precious recognition and treatment of the primary disorder is crucial. The gold standard for the detection of LM is the flexible fiberoptic laryngoscopy that can be performed in wakefulness in mild and less complex cases or under general anesthesia in those most at risk if a multilevel obstruction is suspected [14].

When a state dependent or late onset LM is suspected, DISE may be useful for the detection of the obstruction site and may be effective in diagnosing LM. Moreover, among older children with sleep respiratory symptoms and non-occlusive degree tonsils, DISE may be complementary to laryngoscopy in the diagnostic path. Unfortunately, the severity of obstructive respiratory disorder is not always related to the severity of laryngomalacia or to a specific LM type. This finding is confirmed by a prospective cohort study of children with established OSA, where polysomnography not proved useful in determining the severity of laryngomalacia and in predicting the need for surgery. Among 25 infants with OSA, only 80 per cent underwent supraglottoplasty. Moreover, the apneahypopnea index did not correlate with a high laryngomalacia severity score and it did not differ between those undergoing surgery and those managed conservatively [43]. Nevertheless, other evidences showed that a targeted treatment of laryngomalacia frequently results in a significant improvement of OSAS and associated symptoms [19]. According to our opinion the search for sleep breathing disorders in children with stridor and suspected laryngomalacia is crucial, since several literature data found that children with LM and sleep apneas] have more symptoms and risk of complications such as impaired psychomotor development $[20,21]$. We believe that performing a Polysomnography [PSG] among children with stridor is recommended, since a pulse oximetric screening is often not sufficient in infants with suspected apneas associated with LM. Central apneas are in fact often present and may be normal at this age but they cannot be identified without a PSG. Recent guidelines on obstructive sleep disordered breathing in 1- to 23-month-old children, suggest that the therapeutic approach for young children with OSAS should be individualized according to age, etiology and severity and tools as of nasopharyngoscopy or DISE may be use to decide the type and appropriate sequence of interventions [9].

\section{Conclusion}

Concluding also according to our experience, an accurate endoscopic diagnosis is essential to ensure targeted treatment of both OSAS and laryngomalacia.

\section{Competing Interests}

The authors declare that they have no competing interests.

\section{Funding}

Not applicable.

\section{Authors' Contributions}

MG performed the literature review. GR coordinated the writing group. All authors critically reviewed the manuscript, read and approved the final version.

\section{Acknowledgement}

Not applicable.

\section{References}

1. Ayari S, Aubertin G, Girschig H, Van Den Abbeele T, Mondain M (2012) Pathophysiology and diagnostic approach to laryngomalacia in infants. Eur Ann Otorhinolaryngol Head Neck Dis 129(5): 257-263.

2. Thompson DM (2007) Abnormal sensorimotor integrative function of the larynx in congenital laryngomalacia: A new theory of etiology. Laryngoscope 117 (114):1-33.

3. Bibi H, Khvolis E, Shoseyov D, Ohaly M, Ben Dor D, et al. (2001) The prevalence of gastroesophageal reflux in children with tracheomalacia and laryngomalacia. Chest 119(2): 409-413.

4. Olney DR, Greinwald JH, Smith RJ, Bauman NM (1999) Laryngomalacia and its treatment. Laryngoscope 109(11):1770-1775.

5. Hadfield PJ, Albert DM, Bailey CM, Lindley K, Pierro A (2003) The effect of aryepiglottoplasty forlaryngomalacia on gastro-oesophageal reflux. Int J Pediatr Otorhinolaryngol 67: 11-14.

6. Carter J, Rahbar R, Brigger M, Chan K, Cheng A, et al. (2016) International Pediatric ORL Group (IPOG) laryngomalacia consensus recommendations. Int J Pediatr Otorhinolaryngol 86: 256-261.

7. RB Mitchell, E Call, J Kelly (2003) Ear, nose and throat disorders in children with Down syndrome, Laryngoscope 113: 259-263.

8. RB Mitchell, E Call, J Kelly (2003) Diagnosis and therapy for airway obstruction in children with Down syndrome, Arch. Otolaryngol. Head Neck Surg 129: 642-645.

9. Kaditis AG, Alonso Alvarez ML, Boudewyns A, Abel F, Alexopoulos EI, et al. (2017) ERS statement on obstructive sleep disordered breathing in 1- to 23-month-old children. Eur Respir J 50(6). pii: 1700985.

10. Zalzal GH, Anon JB, Cotton RT (1987) Epiglottoplasty for the treatment of laryngomalacia. Ann Otol Rhinol Laryngol 96(1 Pt 1): 72-76.

11. Holinger LD, Konior RJ (1989) Surgical management of severe laryngomalacia.Laryngoscope 99: 136-142.

12. Hoff SR, Schroeder JW, Rastatter JC, Rastatter JC, Holinger LD (2010) Supraglottoplasty outcomes inrelation to age and comorbid conditions. Int J Pediatr Otorhinolaryngol 74: 245-249.

13. Lima TM, Goncalves DU, Goncalves LV, Reis PA, Lana AB, et al. (2008) Flexible nasolaryngoscopy accuracy in laryngomalacia diagnosis. Braz J Otorhinolaryngol 74: 29-32.

14. Sivan Y, Ben-Ari J, Soferman R, DeRowe A (2006) Diagnosis of laryngomalacia by fiberoptic endoscopy: awake compared with anesthesia-aided technique. Chest 130: 1412-1418.

15. Ng DK, Chan CH (2013) A review of normal values of infant sleep polysomnography. Pediatr Neonatol 54(2): 82-87.

16. Berry RB, Budhiraja R, Gottlieb DJ, Gozal D, Iber C, et al. (2012) Rules for scoring respiratory events in sleep: update of the 2007 AASM Manual for the Scoring of Sleep and Associated Events. Deliberations of the Sleep Apnea Definitions Task Force of the American Academy of Sleep Medicine. J Clin Sleep Med 8(5): 597-619.

17. Richter GT, Rutter MJ, de Alarcon A, Orvidas LJ, Thompson DM (2008) Late onset laryngomalcia: a variant of disease. Arch Otolaryngol Head Neck Surg 134: 75-80. 
18. Amin MR, Isaacson G (1997) State-dependent laryngomalacia. Ann Otol Rhinol Laryngol 106: 887-890.

19. Digoy GP, Burge SD (2014) Laryngomalacia in the older child: clinical presentations andmanagement. Curr Opin Otolaryngol Head Neck Surg 22(6): 501-505.

20. Thevasagayam M, Rodger K, Cave D, Witmans M, El-Hakim H (2010) Prevalence of laryngomalacia in children presenting with sleepdisordered breathing. Laryngoscope 120(8): 1662-1666.

21. Goldberg S, Shatz A, Picard E, Anat Shatz, Elie Picard, et al. (2005) Endoscopic findings in children with obstructive sleep apnea: effects of age and hypotonia. Pediatr Pulmonol 40(3): 205-210.

22. Holinger LD, Konior RJ (1989) Surgical management of severe laryngomalacia. Laryngoscope 99: 136-142.

23. Olney DR, Greinwald JH, Smith RJ, Bauman NM (1999) Laryngomalacia and its treatment. Laryngoscope 109(11): 1770-1775.

24. Hysinger EB (2018) Laryngomalacia, Tracheomalacia and Bronchomalacia. Curr Probl Pediatr Adolesc Health Care 48(4): 113118.

25. Van der Heijden M, Dikkers FG, Halmos GB (2015) The groningen laryngomalacia classification system based on systematic review and dynamic airway changes. Pediatr Pulmonol 50(12): 1368-1373.

26. Sivan Y, Ben-Ari J, Soferman R, DeRowe A (2006) Diagnosis of laryngomalacia by fiberoptic endoscopy: Awake compared with anesthesia-aided technique. Chest 130(5): 1412-1418.

27. Boudewyns A Van de Heyning P, Verhulst S (2017) Drug-induced sedation endoscopy in children $<2$ years with obstructive sleep apnea syndrome: upper airway findings and treatment outcomes. Eur Arch Otorhinolaryngol 274(5): 2319-2325.

28. Ulualp SO, Szmuk P (2013) Drug-induced sleep endoscopy for upper airway evaluation in children with obstructive sleep apnea. Laryngoscope 123: 292-297.

29. Durr ML, Meyer AK, Kezirian EJ, Rosbe KW (2012) Drug-induced sleep endoscopy in persistent pediatric sleep-disordered breathing after adenotonsillectomy. Arch Otolaryngol Head Neck Surg 138: 638-643.

30. Boudewyns A, Van de Heyning P, Verhulst S (2017) Drug-induced sedation endoscopy in children $<2$ years with obstructive sleep apnea syndrome: upper airway findings and treatment outcomes. Eur Arch Otorhinolaryngol 274(5): 2319-2325.

\section{ISSN: 2574-1241}

DOI: $10.26717 /$ BJSTR.2020.26.004331

Giampaolo Ricci. Biomed J Sci \& Tech Res



Commons Attribution 4.0 License

Submission Link: https://biomedres.us/submit-manuscript.php
31.Zafereo ME, Taylor RJ, Pereira KD (2008) Supraglottoplasty for laryngomalacia with obstructive sleep apnea. Laryngoscope 118: 18731877.

32. Iglauer S (1922) Epiglottidectomy for the relief of congenital laryngeal stridor with report of a case. Laryngoscope 32: 56-59.

33. Hasslinger F (1928) Diagnostik and therapie des stridor conenitus. Z Hals NasenOhrenheilkd 21: 223-235.

34. (1994) Indications and standards for use of nasal continuous positive airway pressure (CPAP) in sleep apnea syndromes. American Thoracic Society. Official statement adopted March 1944. Am J Respir Crit Care Med 150(6 Pt 1): 1738-1745.

35. Massa F, Gonsalez S, Laverty A, Wallis C, Lane R (2002) The use of nasal continuouspositive airway pressure to treat obstructive sleep apnoea. Arch Dis Child 87(5): 438-443.

36. Marcus CL, Ward SL, Mallory GB, Rosen CL, Beckerman RC, et al. (1995) Use of nasal continuous positive airway pressure as treatment of childhood obstructive sleep apnea. J Pediatr 127(1): 88-94.

37. Marcus CL (1999) Advances in management of sleep apnea syndromes in infants and children. Pediatr Pulmonol Suppl 18: 1881-189.

38. May JG, Shah P, Lemonnier L, Bhatti G, Koscica J, et al. (2011) Systematic review of endoscopic airway findings in children with gastroesophageal reflux disease. Ann Otol Rhinol Laryngol 120: 116-122.

39. Hartl TT, Chadha NK (2012) A systematic review of laryngomalacia and acid reflux. Otolaryngol Head Neck Surg 147: 619-626.

40. Carter J, Rahbar R, Brigger M, Chan K, Cheng A, et al. (2016) International Pediatric ORL Group (IPOG) laryngomalacia consensus recommendations. Int J Pediatr Otorhinolaryngol 86: 256-261.

41. DGozal, L Kheirandish-Gozal (2007) Neurocognitive and behavioral morbidity in children with sleep disorders, Curr. Opin. Pulm. Med 13: 505-509.

42. Tauman R, Gulliver TE, Krishna J, Montgomery-Downs HE, O’Brien LM, et al. (2006) Persistence of obstructive sleep apnea syndrome in children after adenotonsillectomy. J Pediatr 149: 803-808.

43. Weinstein JE, Lawlor CM, Wu EL, Rodriguez KH (2017) Utility of polysomnography in determination of laryngomalacia severity. Int Pediatr Otorhinolaryngol 93: 145-149.

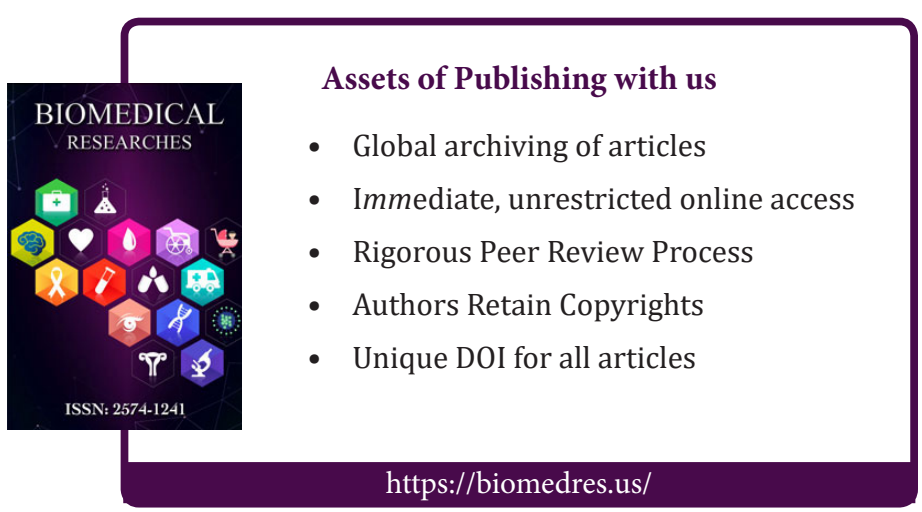

